\title{
La loi de Baumol ou la maladie des coûts dans le système
}

\author{
Jochen Hartwig 1, a,b et Hagen Krämer \\ a Faculté des sciences économiques, Université technique de Chemnitz, Allemagne; ${ }^{b}$ KOF Swiss Economic Institute, EPF Zurich, Suisse; \\ ${ }^{c}$ Faculté des sciences économiques, Université des sciences appliquées de Karlsruhe - Technique et économie, Allemagne
}

\begin{abstract}
Il y a 50 ans, l'économiste William J. Baumol a observé un phénomène dont il a tiré une théorie: les services renchérissent plus vite que les biens matériels. Le présent article montre que la théorie appelée «loi de Baumol» ou maladie des coûts reste très pertinente tant sur le plan théorique que pratique et concerne aussi la manière de gérer les coûts croissants des prestations de santé à l'avenir.
\end{abstract}

En 1967, l'économiste-chercheur de Princeton, William J. Baumol, publia dans l'American Economic Review un article intitulé «The Macroeconomics of Unbalanced Growth: The Anatomy of Urban Crisis» [2]. Cet article a introduit dans la littérature un concept, connu sous le nom de «loi de Baumol» ou maladie des coûts², qui aide à comprendre pourquoi les services renchérissent plus que les biens matériels dans les secteurs de la santé et du social, de la formation, du commerce, de l'art et de la culture et dans de nombreux autres domaines.

Dans l'hypothèse que les salaires (à qualification égale) s'alignent à long terme dans tous les secteurs, Baumol a prédit que les coûts salariaux unitaires et donc les prix de la plupart des services augmenteront plus vite que ceux des produits industriels. Cela voudrait donc dire que certains services renchérissent tellement au fil du temps par rapport aux biens industriels qu'ils en deviennent inabordables.

\section{Les services et la maladie des coûts}

Avec son collègue William G. Bowen, William Baumol soulignait déjà en 1965 les conséquences importantes liées au manque systématique de gain de productivité dans le domaine de l'art [4]. Le «rendement par heurepersonne» d'un violoniste qui interprète un quatuor de Schubert ne peut pas être augmenté sans perte de qualité. Il en va de même pour une représentation de théâtre, étant donné qu'on ne peut pas augmenter la productivité d'Henri IV de Shakespeare, en réduisant simplement le nombre d'acteurs. Dans les années 1960, Baumol et Bowen ont remarqué que les musiciens, acteurs et autres artistes n'étaient pas plus productifs que dans les années 1860, mais qu'ils gagnaient nettement plus que leurs collègues 100 ans plus tôt. Ce gain de revenu, les artistes le devaient à l'augmentation de la productivité du travail dans d'autres secteurs économiques, avant tout dans l'industrie productrice.

La "productivité» des violonistes n'a pas augmenté en 100 ans, contrairement à leurs salaires. C'est pourquoi les services culturels deviennent relativement chers.

Comme les salaires ont augmenté en vertu d'une hausse continuelle de la productivité, les orchestres et théâtres ont été obligés d'aligner les salaires de leurs employés, s'ils ne voulaient pas les voir émigrer vers d'autres secteurs. L'augmentation de ces coûts, et donc la hausse des coûts salariaux unitaires, ne pouvant pas être compensée par une augmentation de la productivité, se traduit par une hausse continuelle du prix des billets. L'art et la culture souffrent d'une maladie des coûts chronique.

Dans son article de 1967, William Baumol a généralisé ces réflexions en les transposant à tous les secteurs économiques dans lesquels les augmentations de la productivité sont sporadiques ou inexistantes. Il a formalisé ses idées à l'aide d'un modèle de croissance néoclassique simple distinguant entre secteurs progressifs et secteurs archaïques. Parmi ces derniers figurent notamment le secteur de la formation et de l'éducation, celui de la santé, de la sécurité ainsi que de l'administration publique, de l'art et de la culture, de l'hôtellerie et de la restauration, de l'artisanat et du logement. Ces secteurs archaïques sont donc principalement ceux des services à la personne. Ils se caractérisent d'une 
manière générale par le fait qu'il n'est pas possible de les automatiser; ce qui explique par ailleurs pourquoi les gains de productivité dans le secteur des services sont nettement plus faibles par rapport au secteur industriel: un fait empiriquement établi pour beaucoup d'économies avancées (cf. [5], p. 99-101).

\section{Le système de santé aussi est un secteur} "archaïque", en raison des nombreux services à la personne. Les augmentations de coûts sont donc inévitables.

En suivant l'évolution des prix de différents biens et services en Allemagne par rapport à l'indice général des prix à la consommation, la figure 1 montre que les divergences prédites par Baumol sur l'évolution des coûts et prix de biens produits industriellement ou de services sont justes. De 1990 à 2016, les prix pour les prestations de formation ont globalement augmenté $50 \%$ plus vite que les prix à la consommation. Les prix pour les services de coiffure - l'exemple parfait pour un service sans véritable potentiel d'augmentation de la productivité - ont augmenté $20 \%$ plus vite que la moyenne. Pour les prestations de santé, le taux d'augmentation des prix dépasse d'environ 15\% la moyenne de tous les prix. La même chose vaut pour les dépenses au restaurant $(+11 \%)$. Les prix pour les denrées alimentaires, les véhicules neufs et les vêtements par contre sont restés inférieurs à l'augmentation générale des prix. L'importante baisse relative des prix pour les appareils de divertissement et de communication (télévisions, téléphones mobiles, etc.) est particulièrement frappante. La loi de Baumol décrit donc un phénomène réel.

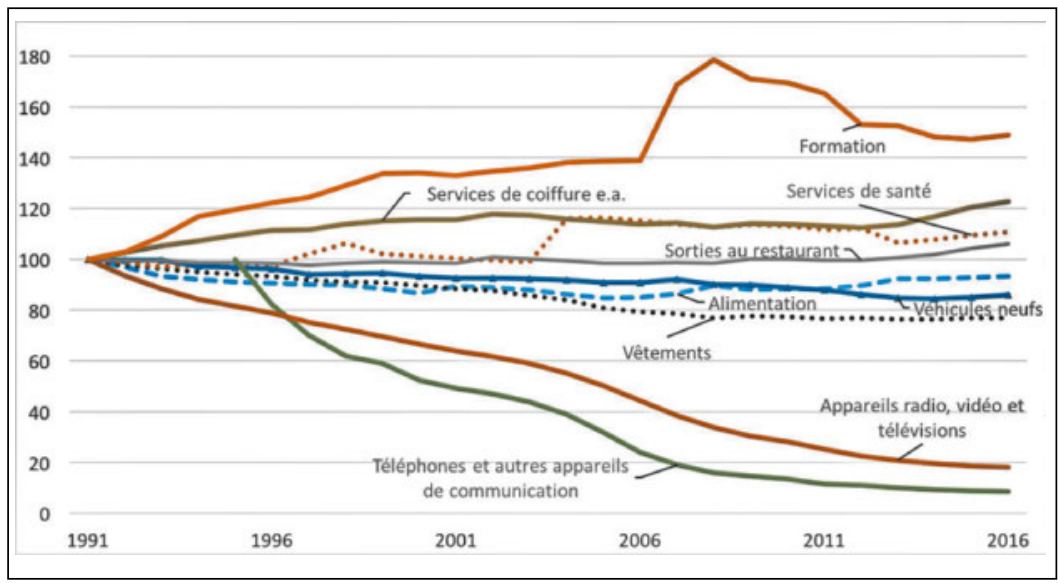

Evolution des prix de différents biens et services par rapport à l'indice général des prix à la consommation.

\section{Ne faudrait-il pas tenir compte de la meilleure qualité?}

Souvent, il est fait état des importantes difficultés qui persistent pour la mesure de la productivité des services, malgré les progrès réalisés par la statistique officielle. Ainsi, on demande que la qualité des services soit davantage prise en compte dans la mesure. Si les améliorations de la qualité étaient comptabilisées comme des augmentations de productivité, à l'instar d'autres domaines de la statistique, on obtiendrait des taux d'augmentation de la productivité plus élevés pour les services.

Même si cette remarque sur le manque de prise en compte de la qualité est justifiée, elle ne permet pas de remettre en question la loi de Baumol. Bien évidemment, les améliorations de la qualité apportent un avantage supplémentaire au bénéficiaire d'un service. Cela ne change cependant rien au fait que les coûts, même de services qualitativement améliorés, augmentent continuellement et que les consommateurs doivent payer toujours plus pour ces (meilleurs) services.

Les services dont le prix a augmenté restent en principe abordables car les revenus augmentent et les prix des biens industriels baissent.

Prenons pour exemple une nouvelle forme de traitement médical pour une maladie potentiellement mortelle qui coûte le double du traitement proposé jusqu'à présent (cf. [6], p. 82-87). Si la productivité est mesurée comme le quotient de la durée de traitement par patient, la productivité des services mesurée de façon conventionnelle peut éventuellement demeurer constante. Si cela permet d'augmenter significativement les chances de survie du patient, le bénéfice de cette prestation médicale augmente bien sûr considérablement, ce qui justifie aussi son prix plus élevé. Si l'on tient compte de cette augmentation de la qualité dans l'indice de productivité, cela se traduit par une productivité des services plus élevée. Il faut cependant financer les coûts plus élevés d'une manière ou d'une autre. Le problème des coûts reconnu par Baumol ne disparaît donc pas, même en tenant compte des aspects de qualité.

\section{Les revenus augmentent, mais sont répartis de façon inégale}

Les augmentations de prix engendrées par la maladie des coûts sur les services à faible productivité n'impliquent pas forcément que ces services ne soient plus 
demandés. En effet, la croissance générale des revenus liée à l'augmentation de productivité peut compenser l'impact de la maladie des coûts. Les consommateurs peuvent s'offrir des services devenus plus chers car leur revenu augmente et, comparativement, les prix qu'ils doivent payer pour les biens industriels baissent (cf. [7]).

Cela soulève toutefois deux autres problèmes majeurs sur le plan de la distribution:

- La croissance des revenus rendue possible par les gains de productivité doit se répartir uniformément entre tous les membres de la société. A défaut de cette évolution, les groupes sociaux qui ne profitent pas de la croissance générale des revenus ne pourront plus se payer certains services.

- D'innombrables services que l'on peut attribuer aux secteurs archaïques (santé, social, formation) sont mis à disposition par les pouvoirs publics. Un renchérissement continuel de ces services implique une mise à disposition de parts toujours plus importantes du budget de l'Etat ou une augmentation des impôts et des cotisations sociales. Il est évident que cela provoquera des conflits liés à la répartition.

Si les classes de revenu inférieures ne profitent pas ou peu de la croissance des revenus, de nombreux services ne seront plus à leur portée.

Pour résumer, il est possible d'affirmer que la croissance générale des revenus peut être un moyen pour gérer la maladie des coûts. Sans mesures complémentaires, la croissance des revenus ne permet cependant pas de guérir la maladie des coûts. Le problème des coûts persiste donc sur le fond.

\footnotetext{
3 Dans le paysage

scientifique suisse, la loi de Baumol est par contre fermement établie. Elle compte ainsi parmi l'un des déterminants des dépenses, tant dans le modèle prévisionnel à court terme des dépense de santé du Centre de recherches conjoncturel les de l'EPF Zurich (voir [13]) que dans le modèle prévisionnel à long term de l'Administration fédérale des finances (voir [14]). Colombier [15] récemment rendu attentif aux incompatibilités entre la loi de Baumol et les règles budgétaires étatiques telles que le «frein à l'endettement».
}

\section{Un secteur à faibles salaires est-il la solution?}

Une toute autre solution à la maladie des coûts serait de transformer les secteurs des services «archaïques» en secteurs à faibles revenus, dans lequel les salaires augmenteraient en fonction du taux de productivité plus faible. Ainsi, les coûts salariaux unitaires dans les secteurs archaïques cesseraient d'augmenter plus vite que ceux des secteurs à forte productivité. La maladie des coûts ne se déclarerait même pas. Mais cela signifierait que les employés, à qualification égale, ne toucheraient pas le même salaire suivant leur secteur d'activité. Des réglementations étatique et professionnelle accompagnées de mesures de pilotage devraient alors être introduites pour empêcher que les salariés moins bien payés quittent le sec- teur archaïque. Cela équivaudrait à la fin de la liberté sur le marché du travail, du libre choix de la profession et du principe de la libre concurrence.

Les tentatives de contrôler les coûts de la santé sont jusqu'ici restées infructueuses et montrent que la véritable raison de l'augmentation des coûts n'a pas encore été comprise.

\section{Conclusions économiques et socio- politiques}

De notre point de vue, le concept de la maladie des coûts n'a rien perdu de son actualité. C'est important, car elle touche de nombreux secteurs de services qui sont déterminants pour une bonne qualité de vie. L'un des secteurs dans lequel il faut s'attendre à une poursuite de l'augmentation des coûts salariaux unitaires est celui de la santé. Les mesures appliquées contre la maladie des coûts, comme la tentative de contrôler les coûts dans le système de santé, n'ont pas fait leurs preuves dans beaucoup de pays. Cela démontre aussi que les milieux politiques n'ont pas encore intégré la vraie raison de l'augmentation des coûts des services aux particuliers.

\section{La maladie des coûts en Suisse}

En Suisse, «l'explosion des coûts de la santé» est un des sujets politiques les plus discutés et a fait l'objet de nombreuses initiatives parlementaires. Le Conseil fédéral tente par exemple de maîtriser les coûts en réduisant les prix des médicaments. Il semble donc que le phénomène de la maladie des coûts demeure largement méconnu. C'est regrettable, car Baumol nous apprend que même sans vieillissement de la population, sans nouveaux médicaments onéreux, sans progrès dans la technique médicale et sans tous les autres facteurs prétendument générateurs de coûts, l'augmentation de la part des dépenses de santé du produit intérieur brut se poursuivra. Ce sont des raisons inhérentes au mode de production qui en sont responsables, auxquelles il faut répondre par d'autres mesures que celles

\section{Même sans vieillissement de la population} et sans progrès médical, la part des dépenses de santé rapportée au produit intérieur brut continuera d'augmenter.

qui ont été discutées jusqu'ici. Déjà Hartwig [8] soulignait cet état de fait et estimait qu'en Suisse, les politiciens adeptes du «tout est possible» et certains journalistes peu éclairés étaient en large partie responsables 
du fait que le débat public ignorait tout de l'effet Baumol sur le système de santé. ${ }^{3}$

\section{Des réactions politiques adéquates sont nécessaires}

L'héritage de Baumol réside dans la mise en garde contre des choix politiques erronés. Notamment pour les services publics, les réactions à la maladie des coûts

\section{La méconnaissance de l'effet de Baumol dans le débat public est due aux politiciens adeptes du "tout est possible» et à certains journalistes peu éclairés.}

se sont jusqu'à présent limitées à des restrictions qualitatives et à la tentative d'imposer des plafonnements de coûts. Cela mène dans la mauvaise direction. Combattre les coûts croissants dans les secteurs de la santé et de la formation ou pour d'autres services à la personne ne constitue pas une réponse pertinente à la maladie des coûts. Il faut premièrement l'accepter et, deuxièmement, gérer de façon adéquate les inévitables augmentations de coûts dans les secteurs archaïques. Dans son dernier ouvrage (cf. [6], p. 180-182), Baumol nous rappelle qu'en principe nous pouvons nous payer des services toujours plus coûteux. Mais nous devons nous attaquer aux problèmes de la répartition.

La bonne nouvelle est que nous pouvons nous payer des services toujours plus coûteux, à condition que nous abordions les problèmes de répartition.

Baumol souligne aussi que la maladie des coûts dans le secteur des services touche principalement les personnes les plus démunies (cf. ibidem, p. 59-60 et p. 180182). Fondamentalement, il est juste d'affirmer que notre société pourra se payer à long terme des services toujours plus coûteux, pour autant que la productivité économique globale et donc les revenus continuent de croître. Il ne faut cependant pas ignorer que cette réflexion se base sur une moyenne. Si les classes de revenu inférieures continuent d'être plus ou moins exclues de la croissance générale des revenus, de nombreux services ne seront plus à la portée des plus pauvres.

La dimension sociale de la maladie des coûts réside par ailleurs dans le fait que ce sont notamment les couches défavorisées qui dépendent des services mis à disposition par l'Etat (surtout dans le domaine de la santé, du social et de la formation). Les problèmes de financement qui s'accentueront à l'avenir en raison de la maladie des coûts risquent d'entraîner de nouveaux conflits liés à la répartition. Il ne faut pas les sous-estimer. La maladie des coûts représente donc aussi un grave problème de répartition qui pourra de moins en moins être ignoré sur la durée.

\section{Références}

1 Hartwig J, Krämer H. 50 Jahre Baumol'sche Kostenkrankheit. Wirtschaftsdienst. 2017:97(11):793-800.

2 Baumol WJ. Macroeconomics of unbalanced growth. The anatomy of urban crisis. American Economic Review. 1967;57(3):415-26.

3 Vandermeulen A. A remission from Baumol's Disease: Ways to publish more articles. Southern Economic Journal. 1968;35(2):189-91.

4 Baumol WJ, Bowen WG. On the performing arts: the anatomy of their economic problems. American Economic Review. 1965;55 (1-2):495-502.

5 International Monetary Fund. Uneven growth: Short- and long-term factors. Washington DC; 2015.

6 Baumol WJ. The Cost Disease. Why Computers Get Cheaper and Health Care Doesn't. New Haven CT; 2012

7 Schettkat R, Yocarini L. The shift to services employment. A review of the literature. Structural Change and Economic Dynamics. 212;17(2): 127-47.

8 Hartwig J. Sind unsere gesamtwirtschaftlichen Probleme überhaupt lösbar? KOF Working Paper 112. Zurich (https://doi.org/10.3929/ ethz-a-005104863); 2005.

9 Hartwig J. What drives health care expenditure? Baumol's model of «unbalanced growth» revisited. KOF Working Paper 133. Zurich (https://doi.org/10.3929/ethz-a-005187505); 2006.

10 Hartwig J. Can Baumol's model of unbalanced growth contribute to explaining the secular rise in health care expenditure? An alter native test. KOF Working Paper 178. Zurich (https://doi.org/10.3929/ ethz-a-005502972); 2007.

11 Hartwig J. What drives health care expenditure? - Baumol's model of 'unbalanced growth' revisited. Journal of Health Economics. 2008;27(3):603-23.

12 Hartwig J. Can Baumol's model of unbalanced growth contribute to explaining the secular rise in health care expenditure? An alternative test. Applied Economics. 2010;43(2):173-84.

13 Hartwig J, Sandqvist P. Ein Modell zur Prognose der schweizerischen Gesundheitsausgaben. KOF Analysen. 2015;9(3):109-25.

14 Colombier C. Ausgabenprojektionen für das Gesundheitswesen bis 2060. Working Paper der Eidgenössischen Finanzverwaltung Nr. 19. Bern; 2012

15 Colombier C. Unwanted side effects of Baumol's cost disease on a balanced-budget rule. mimeo; 2017 